\title{
ENSEÑANZA DE LA MICOLOGÍA. UN ENFOQUE REGIONAL INTERDISCIPLINARIO DE INTEGRACIÓN DOCENTE - ASISTENCIAL
}

\section{(Teaching of Mycology. A regional interdisciplinary approach of educational - assistance integration)}

\author{
R. Salim. \\ Cátedra de Micología, Inst. de Microbiología "Dr. Luis C. Verna" \\ Facultad de Bioquímica, Química y Farmacia,Univ. Nacional de Tucumán \\ Ayacucho 491- (4000) S. M. de Tucumán. Argentina \\ E-mail: rqsalim@unt.edu.ar
}

Palabras clave: Enseñanza, integración docente-asistencial regional, Argentina

Key words: Teaching, regional assistance-integration educational model, Argentina.

\section{RESUMEN}

Se describe una propuesta de integración docente-asistencial para la enseñanza de la asignatura Micología a estudiantes de Bioquimica, con un enfoque interdisciplinario y una estrategia metodológica de acercamiento del alumno a la tarea profesional en el ambiente médico - hospitalario.

El objetivo central de esta propuesta de la articulación entre enseñanza y extensión, es procurar en los alumnos la incorporación de contenidos teóricos, adiestramiento y habilidades que pueden adquirirse en mejor forma en la práctica hospitalaria. De este modo se acrecienta el conocimiento práctico, se coteja realidad/teoría, y se incrementa la experiencia de los docentes que no están habituados a trabajar con pacientes hospitalizados, contactando a ambos, docentes y estudiantes, con la realidad socioeconómica y cultural del medio.

La integración docente-asistencial, plantea además una mayor y más profunda vinculación entre la universidad y la comunidad, al abrir un espacio más allá del aula y la posibilidad de prestación cooperativa de servicios altamente especializados.

Se destaca la necesidad de potenciar la difusión de la disciplina entre los profesionales de la salud y se subraya la importancia de la formación interdisciplinaria, multiprofesional y ética, cuyo capitulo más importante y urgente es el de los deberes humanos frente a los demás.

\section{INTRODUCCION}

El presente trabajo es el resultado de una serie de cuestionamientos que, como Profesora Adjunta de la asig-

\section{SUMMARY}

An educational and assistance integration proposal to teach mycology to students of biochemistry, making emphasis on an interdisciplinary and methodological strategy order to integrate the student into the medical-hospital environment is described.

The main purpose of this proposal of teaching extension articulation is to promote in the students the incorporation of theoric contents and the development of training and skills that can be best obtained in the practice, at the hospital level.

Thus the practical knowledge can be increased and reality can be confronted to theory especially the teachers' experience who are not accustomed to work with hospitalized patients, contacting both, teachers and students with the cultural, social and economical reality in the region. ,

The teaching - assistance integration proposes a greater and deeper closeness between the university and the community by opening a space beyond the classroom and the possibility of offering highly specialized cooperative services.

The need to strengthen the diffusion of the discipline among the health proffesionals and the importance of the interdisciplinary, multiproffesional formation is pointed out. The ethical formation is emphasized as one of the most important and urgent chapters mainly thath of the human rights in relation to other people.

natura de Micología que se dicta en la carrera de Bioquímica, surgen al indagar sobre la práctica del ejercicio profesional de los egresados en relación con la disciplina. 
Entre otras incumbencias profesionales, en nuestro país(Argentina), es responsabilidad del profesional Bioquímico el diagnóstico microbiológico de enfermedades micóticas, bacterianas, virológicas y parasitarias, entre otras situaciones profesionales. En el laboratorio de micología se establece el diagnóstico, se confirma la enfermedad y se realiza el monitoreo del curso de la misma ( 9 , $10,12)$.

En los hospitales de nuestra provincia hay sólo dos laboratorios de diagnóstico micológico para la cobertura de una población aproximada de 1.000 .000 de habitantes. Y son muy pocos los bioquímicos dedicados a esta especialidad, a pesar que las micosis han dejado de ser una rareza que interesa sólo a pocos especialistas. En los últimos años la incidencia de estas enfermedades ha aumentado en forma alarmante. Hoy en día representan una importante causa de morbimortalidad $(6,9,12)$.

Se considera que aproximadamente el $2 \%$ de las personas hospitalizadas desarrollan infecciones fúngicas nosocomiales (2). Las micosis superficiales afectan a más del $20 \%$ de la población mundial y las oportunistas están asumiendo una mayor importancia debido a su aumentada incidencia entre los pacientes transplantados, operados, quemados, drogadictos, con SIDA, leucemias, neoplasias, etc. $(6,10,12)$. Sus impactos y consecuencias, indican la necesidad de aumentar la cobertura diagnóstica.

La presentación clínica de las micosis es sumamente inespecífica y carente de signos patognomónicos propios, por lo que se confunden fácilmente con otras patologías. En nuestro medio, al no ser afecciones de denuncia obligatoria, su impacto en salud pública es desconocido. En nuestra experiencia, el médico generalmente piensa en una micosis cuando fracasan los tratamientos para otras enfermedades $(8,13)$. En la mayoría de las veces esta tardanza, puede tener graves o fatales consecuencias para la vida del paciente. Este inadecuado conocimiento de la disciplina impide resolver en buena forma los problemas que se plantean en la práctica cotidiana (10).

Esta situación, que hemos documentado en varios trabajos, agudiza la necesidad tanto de laboratorios como de personal médico especializados, que consideren a la etiología fúngica entre las causas de infección $(7,8,13$, $14,15)$. Es necesario considerar a todos los hongos capaces de crecer a $37^{\circ} \mathrm{C}$, como patógenos potenciales, los que suman más de un centenar de especies de compleja biología (2). Enseñar tal diversidad de aspectos resulta muy complejo, y el problema se agrava si debe realizarse en poco tiempo y lejos del ámbito hospitalario.

Indudablemente el diagnóstico temprano y certero, el tratamiento oportuno y la recuperación del paciente, se logran con una mejor calidad en la atención médica y en la educación sanitaria. Consideramos que el punto clave es optimizar el abordaje de la enseñanza de la Micología y su difusión. Estos dos puntos neurálgicos pueden responder a una estrategia educativa de integración docente - asistencial con un enfoque interdisciplinario $(1,3,4,5)$.

Para abordar adecuadamente el estudio integral de la Micología y para avanzar en los conocimientos, han surgido grupos multidisciplinarios formados por diversos profesionales de diferentes áreas, como la biología molecular, ecología, epidemiología, inmunología clínica, diagnóstico de laboratorio, terapéutica, etc. (9).

Son pocos los cursos universitarios que se dictan y son pocos los espacios abiertos para el análisis, la discusión y la reflexión sobre los temas candentes de la Micología cotidiana. Por ello una de las metas principales de la presente propuesta es cambiar muchos de los prejuicios existentes acerca del interés sanitario de la Micología y potenciar su difusión entre los profesionales de la salud que se enfrentan a los problemas generados por los hon$\operatorname{gos}(10,12)$.

Como la enseñanza práctica, implica una relación docente-alumno-conocimiento, que conlleva la posibilidad de transformaciones, nos proponemos centrar la atención en los problemas que ofrece la enseñanza de la Micología a los estudiantes de Bioquímica y la revisión de las prácticas pedagógicas instaladas en la Cátedra durante más de tres décadas.

Con el objetivo de mejorar las condiciones de enseñanza-aprendizaje regional, se presenta una propuesta didáctica de integración docente-asistencial con una estrategia metodológica de acercamiento del alumno a la tarea profesional en el ambiente médico-hospitalario.

La estrategia metodológica que se presenta permitirá además: 1) fomentar la educación interdisciplinaria y multiprofesional, 2) potenciar la difusión de la Micología entre los profesionales de la salud, 3) abrir un espacio más allá del aula, que permita cotejar realidad/teoría, 4) incrementar la experiencia de los docentes que no están habituados a trabajar con pacientes hospitalizados, 5) formar graduados conscientes de su rol social y en contacto con el ámbito donde desempeñarán su profesión, 6) contactar a docentes y estudiantes con la realidad socioeconómica y cultural del medio y 7) ampliar y profundizar la integración entre la universidad y la comunidad.

\section{PANORAMA ACTUAL}

En trabajos previos hemos puesto en evidencia el bajo registro de micosis oportunistas y sistémicas que se detectan en nuestro medio. Esta situación puede deberse a que los médicos todavía subestiman la morbimortalidad de las infecciones fúngicas, a un subregistro de su real incidencia, por la falta de reconocimiento de las mismas y/o quizás a la escasa especialización de los profesionales de la salud. 
Creemos que uno de los principales problemas a resolver es el de la formación de estos profesionales .

En la mayor parte de los países la Micología no es reconocida como especialidad. La extensión e intensidad soncedida a su estudio en las Facultades de Ciencias de la Salud carecen de uniformidad (10).

En la Universidad Nacional de Tucumán, la Micología se dicta tanto en la Facultad de Medicina como sn la de Bioquímica.

En la Facultad de Medicina la asignatura se enseia parcializada en temas de programas de diferentes mateias (Microbiología, Dermatología, Neumonología, Clínica $\therefore$ Infecciosas). No existe una asignatura que trate todos os aspectos clínicos de las micosis o un espacio de interración de conocimientos que facilite una visión de la Micología en su totalidad. La mayor información se brinla en la asignatura Microbiología, ubicada en 3er año, cuanlo el alumno aún carece de conocimientos básicos que le sermitan asimilar los conceptos clínicos sobre las micosis. Esta información tiende a ser escasa, tanto por el poco iempo concedido a su tratamiento como a la falta de per;onal docente con conocimientos prácticos del problema. 4 esta segmentación del conocimiento de la disciplina, se ;uman problemas adicionales: falta de integración entre as ciencias básicas y clínicas, una excesiva orientación or especialidades, que favorece una visión parcial e inzompleta de la Micología y la desvinculación de las necesidades de la sociedad.

En la Facultad de Bioquímica, la asignatura está ıbicada en el ciclo profesional de la carrera $\left(5^{\circ}\right.$ año). Su luración es de un cuatrimestre. El programa teórico es muy :ompleto y abarcativo de temas de Micología básica y clíuica. El abordaje de la enseñanza está centrado en el doiente. Las actividades de enseñanza /aprendizaje son las llases magistrales, las clases teórico - prácticas y los traJajos prácticos en el laboratorio. La formación práctica en ispectos básicos de la disciplina es completa y adecuada. ?ero como la asignatura se dicta en el ámbito de la Faculad, alejado del hospital, la formación de neto corte clínico ss inadecuada ya que se basa en el estudio de muestras slínicas de pacientes sin contacto con el mismo ni con el médico de cabecera, repitiendo un modelo didáctico hereJado, de más de tres décadas.

La actitud de los docentes de la Cátedra hacia la enseñanza es conservadora y privilegia la adquisición de zonocimientos (información). Esta actitud conservadora parece corresponderse más con el modelo curricular tradizional en el que los docentes se formaron, que a la formazión y capacitación pedagógica recibida en cursos de posgrado. Hay una franca disociación entre la práctica docente y la extensión, ausencia de retroalimentación permanente entre teoría y práctica y una cierta tendencia a la frondosidad enciclopedista.
El panorama descrito, exige un replanteo crítico de la práctica pedagógica y un cambio muy significativo de apertura a reformas, de revisión de criterios metodológicos y a un nuevo planteamiento de la docencia para el mejoramiento educativo en la Cátedra.

\section{DISEÑO DE LA PROPUESTA Y FUNDAMENTACIÓN TEÓRICA}

Por lo expresado, resulta evidente que en Tucumán es necesario renovar la práctica docente para mejorar la enseñanza, provocar el desarrollo de un nuevo perfil docente y estudiantil y desarrollar un nuevo modelo de enseñanza-aprendizaje de fuerte compromiso social.

La propuesta alternativa de enseñanza que se presenta ha sido diseñada teniendo en cuenta los aspectos que se consideran más relevantes en relación a la disciplina:

1.- Se plantea abordar la enseñanza de la Micología inserta en el ámbito hospitalario, por considerar a este, el único lugar donde ciertas etapas del curso natural de las enfermedades pueden ser estudiadas, por ser el paciente el primer eslabón de esta cadena. La propuesta se diseña a partir de la convicción de que el alumno debe integrarse a la actividad cotidiana en el hospital para vincular diferentes ámbitos de la práctica en terreno (3). Se espera que esta integración estudiantil garantice su acceso a los fundamentos científicos de la disciplina, la articulación teoria/ práctica y el aprendizaje jerarquizado de aquellas conductas que como futuro profesional de la salud no puede ignorar.

2.- Con el desarrollo científico se produce gran cantidad de nuevos conocimientos. Este crecimiento exponencial de la información provoca la continua segmentación de la ciencia médica en subespecialidades cada vez más nuevas y estrechas.

El conocimiento que se adquiere aisladamente tiende a no ser aplicado y en consecuencia es prácticamente olvidado. Para romper la visión fragmentaria de los conocimientos de Micología a nivel médico-asistencial, es necesario un enfoque interdisciplinario y totalizador en el que cada disciplina aporte una parte de la solución para lograr un entendimiento integral, facilitando la percepción de la totalidad $(3,5)$. En este modelo no se yuxtaponen las distintas áreas del saber, sino que se articulan los conocimientos de diferentes campos.

Al superar la segmentación de los conocimientos, la enseñanza integrada facilita el desarrollo de la habilidad para resolver problemas y la realización de actividades colaborativas. Cada disciplina aporta una parte de la solución para lograr un entendimiento integral de la problemática tratada. 
3.- La formación de profesionales de la salud es hoy más compleja, tanto por el gran desarrollo de la ciencia y de la técnica como por la existencia de nuevos valores sociales y la nueva estructuración en el mercado del trabajo. Abrir un espacio más allá del aula es de capital importancia para que los docentes tengan un real, efectivo y continuo contacto con el medio laboral. Se crea de este modo un espacio en el que se puede cotejar realidad/teoría y a la vez incrementar la experiencia de los docentes que no están habituados a trabajar con pacientes hospitalizados.

4.- El laboratorio de micología juega un rol de importancia cada vez mayor en la recuperación, aislamiento e identificación de los hongos. En él se establece el diagnóstico, se confirma la enfermedad y se realiza el monitoreo del curso de la misma $(9,10,12)$. Sin embargo, en nuestro medio y en el país, la escasez de centros de salud que cuentan con laboratorios de diagnóstico micológico es indicativo del poco interés sanitario que despierta la disciplina.

El éxito del laboratorio depende en gran medida de la recolección del material clínico apropiado y de la elección del sitio correcto para la toma de dicho material. Tanto la extracción como el manejo de la muestra clínica son elementos fundamentales en la certeza del diagnóstico (6). Si el médico no está alerta sobre los síntomas y signos de la infección micótica, puede no obtener una muestra clínica apropiada o enviar el material al laboratorio en condiciones inadecuadas. El diagnóstico entonces puede no ser el adecuado.

5.- Es necesario insistir entonces en que la eficiencia con la que es evaluado un paciente y la eficacia de su tratamiento, dependen de la calidad de la comunicación entre los participantes del equipo de salud. El laboratorio no debe convertirse en una isla, los micólogos no deben aislarse de la práctica clínica (6) y el médico debe informar si sospecha una micosis. Es necesaria la inmediata comunicación médico- micólogo ante los primeros indicios de la presencia de un hongo patógeno, para resolver rápidamente el significado clínico del aislamiento y la necesidad de adicionales evaluaciones .

En nuestra propuesta de enseñanza, la integración docente-asistencial se plantea como estrategia para favorecer la relación de docentes y estudiantes con el paciente, el médico y el ambiente hospitalario. Esta inserción intensificaría el entrenamiento práctico del alumno, favoreciendo su acercamiento a la tarea profesional. Al mismo tiempo se pretende fortalecer la formación de graduados conscientes de su rol social y en contacto con la realidad socioeconómica y cultural del medio.
6.- Para potenciar en nuestro medio, los conocimientos y la difusión de la disciplina entre los profesionales de la salud, consideramos fundamental impulsar la participación de los diferentes especialistas y docentes en ateneos, juntas de interconsultas u otras actividades que posibiliten la discusión de los aspectos clínicos y de laboratorio de las micosis. Este enfoque interdisciplinario posibilitará además consolidar equipos de salud altamente calificados.

Es de especial importancia que la educación interdisciplinaria y multiprofesional planteada, cobra una especial relevancia cuando se quiere producir como en nuestro caso, un efecto de integración de conocimientos y aplicación a situaciones reales (3). Creemos que el modelo educativo que estamos presentando podrá, asimismo garantizar una tarea asistencial del más alto nivel de calidad, causando un fuerte impacto sobre las condiciones de salud de la población.

7.- Aparentemente en nuestro medio el interés sanitario por la Micología no se aprecia o es muy bajo por lo que es necesario ampliar la vigilancia, el control y la denuncia de las micosis, para conocer su impacto real en nuestra región. La implementación de esta nueva práctica de enseñanza, puede contribuir a cambiar muchos de los prejuicios existentes acerca de la importancia de la disciplina entre los profesionales de la salud que se enfrentan cotidianamente a los problemas generados por los hongos.

8.- Este proyecto implica la necesidad de involucrar en el proceso formativo de los alumnos un fundamento ético y una actitud de fuerte compromiso e inserción en la sociedad. Al mismo tiempo consideramos necesario que el docente se adapte a un rol diferente, abandonando el papel de inagotable fuente de información.

9.- Este modelo supone, desde la docencia y la investigación universitaria a la difusión de conocimientos en la sociedad, como devolución del sustento que de la sociedad recibe (11). Desde este punto de vista, la devolución consiste en servicios asistenciales de alta calidad y excelencia, subrayando la importancia de la formación ética, cuyo capítulo más importante y urgente es el de los deberes humanos frente a los demás.

10.- En un mundo que avanza sin pausa hacia la interacción institucional, es imposible mantener el aislamiento que ha caracterizado a muchas universidades. Es necesario conformar un verdadero sistema en el cual diferentes instituciones se relacionen para intercambiar informaciones, saberes y experiencias. Esto permite, a nuestro criterio, abrir un espacio de cooperación interinstitucional 
para utilizar con eficiencia los recursos, resolver los problemas que socavan la salud y mejorar la calidad de vida.

La extensión le confiere a la universidad el rol de transformación social, contribuyendo a una mejor calidad de vida de los habitantes, retribuyendo a la sociedad con respuestas efectivas que reafirmen la principal función: generar conocimientos y propuestas superadoras del atraso yla marginalidad (11).

En este sentido, uno de los primeros pasos sería la integración activa de la universidad a las tareas que desarrollan las otras instituciones en el medio, mediante iniciativas como la que proponemos en este proyecto de inserción de la enseñanza de la Micología en el hospital. Esta cooperación interinstitucional cubriría las expectativas de la comunidad, sobre todo de los contribuyentes que sostienen con sus impuestos este sistema universitario (11). Por lo tanto, la búsqueda y adopción de estrategias participativas que logren acercar a los docentes con la sociedad, deben ser incesantes y un verdadero motivo de preocupación para las autoridades universitarias.

En concreto, la presente propuesta plantea, mediante el asistencialismo y la transferencia de servicios, ampliar y profundizar la vinculación entre la universidad y la sociedad (16). Se pretende desarrollar un nuevo perfil docente y estudiantil así como un nuevo modelo de enseñanza- aprendizaje de fuerte compromiso social.

Cada cátedra tiene la oportunidad de incluirse en la extensión, donde los trabajos prácticos pueden constituir un espacio interesante para su concreción. Llevar a cabo un trabajo práctico de laboratorio, o prácticas médicas, demanda de un tipo de docente que transfiere conocimientos y experiencias personales. Con la modificación que proponemos, la renovación de la práctica docente es sustantiva: insertarse en prácticas sociales diversas, compartidas, ayudar a clarificar problemas, relevarlos, elaborarlos, investigar, integrar equipos multidisciplinarios, despertar el interés sanitario por la Micología y la difusión de la importancia que tiene ésta dentro de la Medicina, entre otros.

Creemos que la inserción de la Cátedra de Micología en el hospital, como práctica de extensión, nos permitirá revitalizar la práctica docente, repensarla y modificarla. Proponemos un aprendizaje basado en el alumno, basado en la salud, basado en problemas, sistemático y de integración del conocimiento. Al mismo tiempo pretendemos lograr un mejoramiento y aumento de la cobertura diagnóstica.

La calidad ha llegado a ser una de las preocupaciones fundamentales de la Educación Superior y depende de la calidad de su personal, sus programas y sus estudiantes así como de su infraestructura y su entorno académico (16). Consideramos que la introducción de los alumnos de medicina y de bioquímica en el sistema de salud, mediante programas educativos y de formación de recursos humanos interdisciplinarios puede producir un marcado efecto de integración y aplicación de conocimientos a situaciones reales promoviendo el ejercicio del trabajo interdisciplinario.

\section{ASPECTOS ORGANIZATIVOS}

Esta propuesta puede concretarse mediante un Convenio de Cooperación Asistencial con el Sistema Provincial de Salud.

La universidad aportaría su planta de personal docente y su compromiso será el de la atención de los análisis micológicos de los pacientes internados y ambulatorios de los servicios de infecciosas, neumonología, dermatología, desnutrición, clínica quirúrgica, quemados, otorrinolaringología, cuidados intensivos y otros. Por los servicios de atención a pacientes ambulatorios se cobraría un bono contribución mínimo para solventar en parte los gastos que demanda el servicio.

El Sistema de Salud por su parte se comprometería a permitir el tránsito de los alumnos de la Cátedra de Micología por los citados servicios, la toma de muestras clínicas a pacientes internados y ambulatorios bajo supervisión y la participación en seminarios y ateneos conjuntamente con los médicos y residentes de los distintos servicios así como presenciar las sesiones de interconsultas profesionales y el análisis de casos clínicos.

\section{REFLEXIONES FINALES}

La presente propuesta constituye la búsqueda de herramientas que mejor se adecuen a la disciplina y a la situación real en que se desarrolla nuestra práctica docente; no constituye una respuesta acabada ni agota la problemática, pero sí plantea un punto de partida para ponerla a prueba y evaluarla concretamente en la práctica cotidiana. Muchas vecès se legaliza una determinada concepción, que al no haber sido analizada críticamente se cree que es la única. Lo que es importante en definitiva, no es el cambio en sí mismo, sino que este pueda producir mejoras con relación a la actual situación de enseñanza descontextualizada de la realidad.

Se pretende otorgar una mayor significatividad a los contenidosy un accionar interdisciplinario que permita el trabajo cooperativo dejando un amplio espacio abierto a la participación de los estudiantes, lo que redundará en una mayor profesionalización docente y una mejor calidad educativa.

Hoy, más que nunca, es necesario defender la calidad académica y resignificar la presencia de la universidad en el medio con un accionar de alto contenido ético y de fuerte compromiso social. 


\section{REFERENCIAS}

1.- Bligh, J. (1995). Problem-based learning in medicine: An introduction. Post grad Med. J. 71:323-36

2.- Ezkurra. P.; Bikandi, J. \& Quindós, G. (1993). Estado actual y perspectivas futuras de la Micologia Médica. Rev. Iber. Micol. 10:29

3.- Galli, A. (1991). Universidad y Salud: Integración DocenteAsistencial. Programa de Formación Docente Pedagógica. Public. de la Organización Panamericana de la Salud. Washington. Pág. 5054

4.- Ghali, W.; Saitz, R.; Eskew, A.; Gupta, M. Quan, H.; Hershman W.Y. (2000). Successful teaching in evidence-based medicine. Med. Educ. 34:18-22

5.- http://www.insa.edu./tam/cpbl/>. Integración interdisciplinaria

6.- Koneman,N. et al., (1997). Diagnóstico Microbiológico. Third Ed. Edit. Panamericana. pp. $657-660$

7.- Laborda R. \& Salim R. (1995). Detección de especies de Nocardia aisladas de pacientes con compromiso pulmonar crónico en Tucumán (Argentina). Boletín Micológico 10:33-36

8.- Laborda, R.; Salim R. \& Boente M. del C. (1998). Zigomicosis rino-sinuso-orbital post-traumática. Boletín Micológico Vol 13: 1115

9.- López, R.; Méndez, L.; Hernández, F.; Castañón R. (1995). Micología Médica. Procedimientos para el diagnóstico de laboratorio. Ed. Trillas. México. pp. 7-8
10.- Negroni, R. (1990). Enseñanza de la Micología Médica, para estudiantes de Medicina y Médicos Generales. Rev. Arg. Micología, $13: 17-32$

11.- Ploper, D. (1998), Universidad y Región. Cuadernos del F.O.R.UN. Ed. Univ. Nacional de Tucumán. Vol. 1:23-31

12.- Richardson, M. \& Warnock D. (1997). Fungal Infection. Diagnosis and Management. Second Ed. Blackwell Sc. Edinburgh Pag. 1-10

13.- Salim, R.; Villagra de Trejo C.; Laborda R. (1997). Nocardia asteroides en absceso pulmonar interpretado como de origen tuberculoso: Caso fatal. Boletín Micológico 12: 95-98

14.- Silva, J.; Laborda, R.; Almendro G.; Salim R. (1989). Rhodotorula glutinis y $R$. rubra: agentes de micosis oportunistas en el hombre. Boletín Micológico 4:171-174

15.- Silva, J.; Laborda, R.; Almendro, G. ; Salim R. (1990). Detection of opportunistic yeast pathogens in hospitalized immunocompromised patients. Rev. Lat-amer. Microbiol. 32: 261264

16.- Yapur, M. C.; Buhler, M. \& Salim, R. (1998). Programa de Autoevaluación Institucional. Informe Final. Ed. Rectorado Universidad Nacional de Tucumán. Argentina. pp. 135-146 